\title{
Preventing Privacy Attacks and Cybercrime in the Mobile Internet
}

Abstract for Panel Session

\author{
Simone Fischer-Huebner ${ }^{1}$, Alberto Escudero Pascual ${ }^{2}$, Helena Lindskog ${ }^{3}$ \\ 'Panel Session Chair,Dept. of Computer Science, Karlstad University, Sweden \\ ${ }^{2}$ Panelist, IMIT, IT University, Royal Institute of Technology, Sweden \\ ${ }^{3}$ Panelist, Privacy Manager, Service Network and Applications, Ericsson AB, Sweden
}

\section{INTRODUCTION (Simone Fischer-Huebner)}

In the mobile Internet, personal privacy is increasingly endangered. Personal data such as traffic data, content data, location data and parameters for personalized or context-aware services are exposed at different network sites and can be easily condensed to extensive user profiles. At the same time, computer networks and electronic information may be used for committing cybercrime attacks. This panel session will discuss:

- Privacy risks and cybercrime threats in the Mobile Internet

- Legal approaches for protecting privacy in the Mobile Internet, such as the proposed EU Directive COM (2000) 385 on privacy in electronic communication for regulating the processing of traffic and location data, confidentiality of communication, cookies and spamming will be discussed

- Technical means for privacy protection by data minimization or avoidance (e.g., through flat rate billing or anonymisation of location data), or by informed user consent (e.g., through P3P)

- Conflicting interests and approaches on traffic data retention or preservation by law-enforcement authorities.

The panel session will start with two short presentations that are summarized in the next two sections.

The original version of this chapter was revised: The copyright line was incorrect. This has been corrected. The Erratum to this chapter is available at DOI: 10.1007/978-0-387-35609-9_29 


\section{TECHNICAL AND LEGAL PRIVACY RISKS IN THE NEXT GENERATION INTERNET (Alberto Escudero Pascual)}

The global telecommunication infrastructure will slowly converge toward an integrated packet switched network using the Internet Protocol version 6 as common transport technology. IPv6 satisfies the new requirements of the next generation Internet that includes security, manageability, mobility, and scalability that offers voice, data and multimedia services.

One of the benefits of IPv6 mobility is the capability of a mobile node to be reachable by a fixed identifier (its home network IP address) with independence of the point of attachment to the Internet.

Continuous technological developments have also been reflected upon the legal scene where the proposed EU directive $\operatorname{COM}(2000) 385$ intends to replace Directive $97 / 66 / \mathrm{EC}$ concerning the processing of personal data and the protection of privacy in the telecommunications sector by adapting and updating the existing provisions to new and foreseeable developments in electronic communications services and technologies.

During the panel we will present some of the research results of the PETng Project Privacy Enhanced Technologies in the next generation Internet', as follows:

- In the technical area, after giving an overview of location privacy in mobile Internet and proposed extensions to the mix-net based Freedom Network, we will present how some technical improvements in IPv6 over IPv4 also introduce privacy risks. For example, mobility signaling and addressing information remain unprotected presenting a clear threat for location privacy.

- In the legal arena we will show how traditional legal, regulatory and technical provisions were established with traditional technological environments in mind and we will argue how some initiatives to update traditional powers of investigation involving technology do not always reflect the sensitivities raised by the current technological environment.

- Finally, we will show how the Platform for Privacy Preferences (P3P) can be used to provide a simple, automated way for users to gain more control over the use of their location information. 


\section{USER CONTROL IN MOBILE INTERNET SYSTEMS (Helena Lindskog)}

Personal data and its protection will be the key to the success of the mobile Internet. Much more than in the legacy Internet, the use of mobile, constrained devices will need personalization and context-dependant services.

Cookies, and the opt-in legislation proposed by the European Commission, have lately been widely debated. Cookies can indeed be very privacy-intrusive. They can also be a useful tool to provide convenience for the end user. The discussion, both within research fora, and within political fora, has so far been a bit binary. We welcome a more granular way of arguing, where industry and legal entities work together to first determine which kinds of cookies that are harmful, e.g. third-party persistent cookies with no P3P policy, as opposed to which kinds that really make a difference to the end user's convenience, and as a next step determine how to deal with the problems.

The spam, or unsolicited messaging, problem, which is also under discussion by European privacy legislators, will be a disabler of the future mobile services. Messaging in mobile environments can be SMS, email and MMS (Multimedia Messaging Services). It is crucial that this privacy problem is handled before it arises - or else, we risk loosing a number of potential end users, that will be annoyed by the download cost, the download time, and the time that it takes to delete unwanted messages, in future email and MMS systems.

The protection of the end user must not be at the cost of the protection of the system and its other users, and of society. Safeguards must be taken where the user is or might be a terrorist or another criminal.

A perfect profiles and identity management system will provide the end user with the control - and perception of this control - to add, change and remove personal data, but also with a powerful user-agent that will handle the data conveyance in accordance with the user's privacy and convenience preferences. Usability aspects must be taken into account. Such a system should also handle the publication of content, and the rulesets of this publication, as well as the rulesets for messaging. P3P preferences are also a natural part of such a system.

Privacy, control and trust will bring the users to the mobile services. 